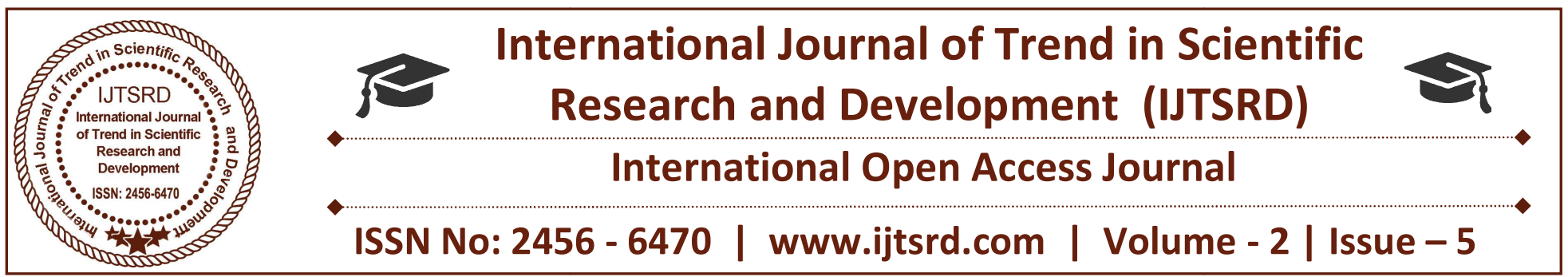

\title{
Identification of Medicinal Plants using Geometric Features of Leaf Image Through SVM
}

\author{
Amrita Arjun Kindalkar ${ }^{1}$, Prof. S. A. Angadi ${ }^{2}$ \\ ${ }^{1}$ Student, ${ }^{2}$ Professor, \\ ${ }^{1,2}$ Department of Computer Science, Visvesvaraya Technological University, \\ Belgaum, Karnataka, India
}

\section{ABSTRACT}

Plants are important to all the living beings. Further in the Indian Science, it is considered as greatest asset and gives rise to a new science called Ayurveda. Some plants provide food some have medicinal value. People instead of curing through medication which is at door step they go behind fastest cure without knowing its side effect. The reason for this is lack of knowledge for identifying medicinal plants. So inorder to avoid this we proposed a computer based technique so that we can identify the plant and give its medicinal values that will help common man to know which are the medicinal plants and also its uses. This paper describes about identifying plants species with the formation of feature set, through computer vision.

Keywords: SVM, Image preprocessing, Feature extraction

\section{INTRODUCTION}

Plants play an important role, they exist in surroundings of human beings called ecosystem, and are very important to the living being. There are some plants which are eatable and are grown above or below the ground whereas there are variety of plants which are found to have medicinal values in Indian system called Ayurveda. These type of plants are different form normal plants. In olden days, people could able to identify the plants which helped them to cure diseases [1]. These plants were grown either on backyard or found on the roadsides. Now it has become difficult for the people to identify the medicinal plants. Many people are unaware of the plants so inorder to identify first thing is to consider leaves to classify them. Leaves can be classified based on different types of features such as shape based or the texture based or colour based [2]. The texture which gives quantitative measure, by the arrangement of pixel in the region which is the statistical approach. There are different (other) types of herbal leaves mainly Thulasi, neem etc. In some case feature can be differentiated based on shape and colour feature. Inorder to classify feature vector of the leaves are classified by classification algorithm like PNN (probabilistic neural network), SVM (support vector machine),k-Nearest neighbour, Principal component analysis (PCA),ANN(Artificial neural network). The main aim is to extract the geometric features of the medicinal leaf.These features are further processed by SVM classifier. After that, the results were used in the classification system. This approach gave improvement in average accuracy for classifying 20 kind of leaf .270 leaves are used for training purpose and 180 leaves per plant for testing purpose. The accuracy obtained was $92.044 \%$.

This paper has 5 sections in section 2 gives related work, section 3 proposed methodologies, in section 4 results and conclusion in section 5 .

\section{RELATED WORK}

Paper [1] has described the classification on the selected plant leaves. Plants are major part in ecosystem and medicines are greatest source of revenue. Due to pollution, deforestation the existences of medicinal plants is low. So there is need to identify and replant them for our future. Here its aim at 
implementing the leaf image using image processing. This software should give the closest match to the query. The proposed algorithm is implemented and the efficiency of the system is found by testing it on 10 different plant species. The software is trained with 100 leaves and tested with 50 leaves. The efficiency of the $92 \%$ is found.

Paper [2] describes about the plants and which are the other kind of feature that can be extracted of leaves. This paper also gives description of various kinds of classifiers that can used for the training and for testing purpose to get the final result.

Paper [3] describes on identification of plants leaf by their shaped based and neural network as classifier. The plants types are analyzed by using different shape techniques such as moment invariant and other centriod radii model, where this system gives a quick and efficient classification of plants species.

\section{III.PROPOSED METHODOLOGY}

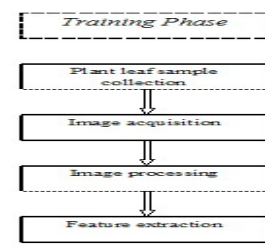

Fig.3.1 Architecture design

Pre-processing:

The input is taken as the image of the leaf. In this step, it removes if there present any external noise in image. The main idea is to find the clear image so that it can be further processed. Then it is converted into grayscale from the color image, which is easy for the extraction. By using the edge detector contour of the leaf is identified.

Feature extraction:

Each leaf has its own feature it can be either shape or its color or texture.

Shape features:

It can be recognized by types of shape based on geometrical feature of leaves.

Area: Area is the number of pixels in the fix space or the region. The area of leaf in a segmented image is the number of white or '1' pixels

Perimeter: number (count)of pixel in the leaf margin.

Major axis length: The line segment joining the lowest point and tip of leaf is the major axis.

Minor axis length: The maximum width, which is perpendicular to the major axis, is the minor axis of a leaf.

Bounding box: It gives the height and width of the leaf

Classifier: The dataset of the leaf stored in the database is trained and tested by the classifier.

Support vector machine: It is one type of classifier used in training and testing process. It performance in multi dimensional space and which is efficient. It map the non- linear to high dimensional and separating the linear data.

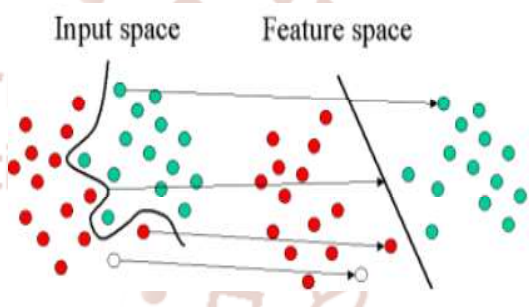

\section{IV.RESULTS}

In the experiments, we used our own dataset by collecting medicinal leafs images. The leaves are captured using the digital camera and set the database. It has 20 medicinal species, atleast 10 samples per species and a total of 450 samples. The sample features are shown in table 1

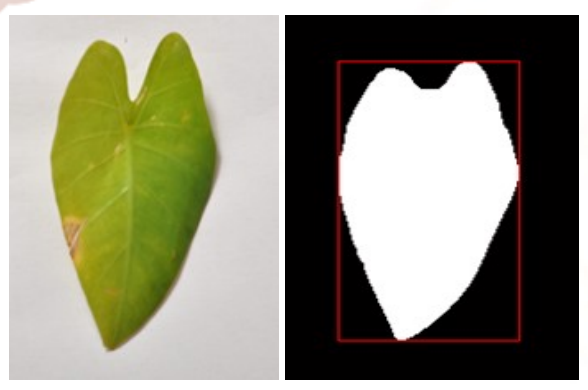

Figure 4.1 Input Colocasia leaf and boundary detected image 
International Journal of Trend in Scientific Research and Development (IJTSRD) ISSN: 2456-6470

Table.1 Feature set for some of the medicinal leaf

\begin{tabular}{|l|l|l|l|l|l|}
\hline \multicolumn{1}{|c|}{ LEAF } & \multicolumn{1}{|c|}{ Area } & Perimeter & Major & Minor & \multicolumn{1}{|c|}{ Bounding box } \\
\hline Colocasia & 15848.00 & 504.48 & 184.70 & 112.46 & $120.00,203.00$ \\
\hline Insulin & 34240.00 & 794.33 & 319.75 & 138.74 & $150.0,342.00$ \\
\hline Keratin & 6753.00 & 420.35 & 166.86 & 53.54 & $1.00,1.00$ \\
\hline Neem & 2398.00 & 241.54 & 96.03 & 33.53 & $104.00,51.00$ \\
\hline Tulsi & 3473.00 & 261.09 & 84.93 & 55.07 & $97.00,62.00$ \\
\hline Hisbiscus & 8639.00 & 3639.00 & 724.99 & 90.32 & $95.00,143.00$ \\
\hline Leamon & 61599.00 & 1007.70 & 368.80 & 213.81 & $222.00,406.00$ \\
\hline Methi & 5526.00 & 317.35 & 122.38 & 59.09 & $137.00,63.00$ \\
\hline Pepper & 54555.00 & 298.17 & 298.17 & 237.54 & $308.00,234.00$ \\
\hline Betel & 43230.00 & 872.22 & 312.19 & 184.6 & $197.00,354.00$ \\
\hline Periwinkle & 17680.00 & 551.64 & 210.24 & 107.88 & $145.00,200.00$ \\
\hline Kepulu & 573.00 & 204.14 & 99.14 & 13.65 & $14.00,35.00$ \\
\hline Hogplum & 9948.00 & 532.24 & 265.84 & 79.35 & $86.00,167.00$ \\
\hline Curry & 1435.00 & 151.00 & 61.23 & 30.42 & $31.00,62.00$ \\
\hline Peelikaner & 9192.00 & 801.47 & 398.85 & 35.96 & $157.00,363.00$ \\
\hline Sambrani & 2762.00 & 199.98 & 69.32 & 52.59 & $8.00,33.00$ \\
\hline Milkweed & 24625.00 & 663.92. & 281.32 & 113.58 & $120.00,285.0$ \\
\hline Panikoorka & 28103.00 & 732.18 & 219.73 & 169.74 & $1.00,1.00$ \\
\hline Parijath & 28383.00 & 730.19 & 236.00 & 160.99 & $2.00,3.00$ \\
\hline Surpin & 9918.00 & 613.41 & 166.61 & 90.92 & $192.00,123.00$ \\
\hline & & & & & \\
\hline
\end{tabular}

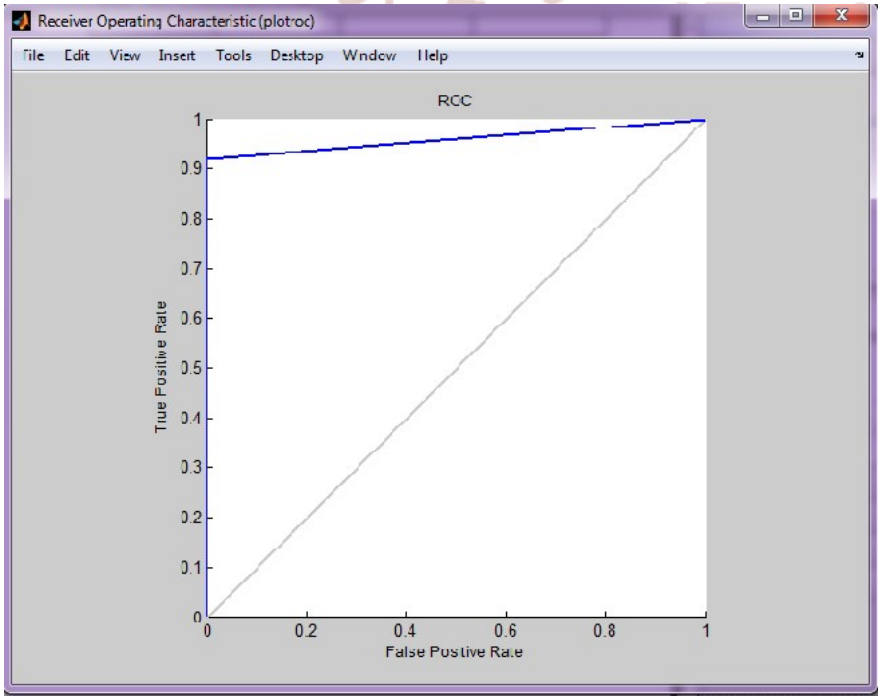

Fig4.2 ROC graph

Here we have used 270 leaves for training and 180 leaves for testing. Finally recognition of medicinal plants using SVM is carried out and is plotted as an ROC graph. The average recognition accuracy obtained is about $92.044 \%$

\section{CONCLUSION}

As plants are important for all the living beings the leaves of the plants help us to cure disease. Here different leaves are been trained and stored in database where it calculate the feature set such has area, perimeter, major axis, minor axis, bounding box etc. It is matched with the input given by the user and gives the geometric feature of that particular leaf.

The proposed methodology helps in identifying the medicinal plants by processing its leaf image. Further the system also displays the medicinal features of the plants. The system will help in automate the traditional knowledge regarding the medicinal values of plants.

\section{REFERENCES}

1. A Gopal, S. Prudhveeswar Reddy and V. Gayatri" Classification for selected medicinal plants leaf using image processings" in proceeding International Conference on Machine Vision and Image Processing (MVIP),2012. .

2. Sapna Sharma, Dr. Chitvan Gupta "A Review of the Plants Recognition Methods and Algorithms" ,International Journal of Innovative Research in Advanced Engineering (IJIRAE) ISSN: 23492163 Issue 6 Volume 2 (June 2015)

3. Jyotismita Chaki, Ranjan Parekh "Plant Leaf Recognition by using Shape based Features and Neurals Networks classifiers" International 
Journal of Advanced Computer Science \& Application, (IJACSA) Vol. 2, No. 10, 2011

4. A. Kadir et al." Performance Improvement of the Leaf Identification System By Using Principal Component Analysis", Internationals Journals of Advanced Sciences and Technology Vol. 44, July, 2012

5. Shitala Prasad, Krishna Mohan Kudiri, \& R.C. Tripathi ," Relative Sub-Image Based Features for the Leaf Recognitions by using Support Vector Machine ", in Proceedings International Conference on Communication, Computing and security ,Pages 343-346, 2013.

6. Anami, Basavaraj S, S. Nandyal Suvarna, \& A. Govardhan. "A combined color, texture \& edge features based on approach for identification and classifications of Indian Medicinal plants." International Journal of Computer Applications, page. 45-51, 2010.
7. Jamil N, Hussin NA, Nordin S \& Awang K. "Automatic Plants Identification: Is Shape the Key Feature?" Procedia Computer Science, vol.74, Pages.36-42, December 2015

8. A Gopal, S. Prudhveeswar Reddy \& V. Gayatri," Classifications of the selected medicinal plants leaf using image processing", in proceedings International Conference on Machine Vision and Image Processing (MVIP),2012.

9. R. Janani, A. Gopal," Identifications of the selected medicinal plants leaf using Image Features and ANN", in proceedings International Conference on Electronic Systems, 2013.

10. Meeta, K., K. Mrunali, P. Shubhada, P. Prajakta, \& B. Neha "Survey on techniques for plant leaf classification." International Journal of Modern Engineering Research (IJMER) Vol no. 2, Pages.538-544, 2012. 